\title{
Números cromosómicos, análisis citogenéticos y primeras descripciones de los cariotipos de Doru lineare y $D$. luteipes (Dermaptera, Forficulidae, Insecta)
}

\author{
Chromosome numbers, cytogenetic analysis and first karyotype \\ descriptions of Doru lineare and D. luteipes (Dermaptera, \\ Forficulidae, Insecta)
}

Gabriela M. Silenzi Usandivaras ${ }^{1 *}$, Aldo R. Andrada1, Valeria A. Páez', María S. Caro ${ }^{1,2}$, Mabel Romero'

1 Fundación Miguel Lillo. San Miguel de Tucumán, Argentina. (*) gmsilenzi@lillo.org.ar

2 Facultad de Ciencias Naturales e Instituto Miguel Lillo, UNT. (T4000JFE) San Miguel de Tucumán, Tucumán. Argentina.

\section{RESUMEN}

Los cromosomas holocinéticos carecen de una constricción primaria y se encuentra mayormente en insectos, entre ellos el orden Dermaptera, comúnmente conocido como "tijeretas". Los integrantes del género Doru son depredadores de plagas en cultivos de maíz, caña de azúcar y algodón, que se distribuyen ampliamente en Sudamérica. En Argentina se encuentran dos especies: D. lineare (Eschs.) y D. luteipes (Scudder). El material se recolectó en la provincia de Tucumán (Argentina). Para teñir los cromosomas se aplicaron técnicas citogenéticas clásicas, así como el método fluorescente DAPI. El análisis comparativo entre $D$. luteipes y $D$. lineare reveló que ambos taxones tienen fórmula haploide $\mathrm{n}=10(9 \mathrm{~A}+\mathrm{XY})$ y $2 \mathrm{n}=20$. Además, se encontraron numerosas irregularidades meióticas y se observaron cromosomas holocinéticos sin constricción primaria que varían gradualmente en tamaño. Se presenta por primera vez información citogenética para $D$. luteipes, se realizan las primeras descripciones de los cariotipos para ambas especies y se discute el posible origen poliploide del género.

Palabras clave - Asociaciones cromosómicas secundarias, cariograma, cromosomas holocinéticos, meiosis, Dermaptera.

Ref. bibliográfica: Silenzi Usandivaras, G. M.; Andrada, A. R.; Páez, V. A.; Caro, M. S.; Romero, M. 2020. "Números cromosómicos, análisis citogenéticos y primeras descripciones de los cariotipos de Doru lineare y D. luteipes (Dermaptera, Forficulidae, Insecta)". Acta zoológica lilloana 64 (2): 116-129. Fundación Miguel Lillo, Tucumán, Argentina. D.O.I.: https://doi.org/10.30550/j.azl/2020.64.2/3

> Recibido: 4 de junio 2020 - Aceptado: 16 de septiembre 2020.

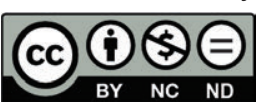




\begin{abstract}
Holokinetic chromosomes lack of a primary constriction and they are mainly found in insects including order Dermaptera commonly known as "tijeretas". Members of the genus Doru are pest predators on corn, sugar cane and cotton crops and they are widely distributed in South America. Two species occur in Argentina: D. lineare (Eschs.) and D. luteipes (Scudder). The material was collected in the province of Tucumán (Argentina). In order to stain the chromosomes, classical staining techniques as well as DAPI fluorescent method were applied. The comparative analysis between $D$. luteipes and $D$. lineare reveals that both taxa have a haploid formula $\mathrm{n}$ $=10(9 \mathrm{~A}+\mathrm{XY})$ and $2 \mathrm{n}=20$. In addition, numerous meiotic irregularities were found and holokinetic chromosomes that gradually vary in size and lack of primary constrictions were observed. Cytogenetic information of $D$. luteipes is presented for the first time, the first karyotype descriptions for both species are performed and it is discussed the possible diploid origin for the genus.
\end{abstract}

Keywords - Secondary chromosomal associations, karyogram, holokinetic chromosomes, meiosis, Dermaptera.

\title{
INTRODUCCIÓN
}

Los cromosomas holocinéticos fueron mencionados por primera vez por HughesSchrader y Ris (1941) en coccidos (Hemiptera; Homóptera). Investigaciones posteriores revelaron que otros insectos tenían como característica este tipo de cromosomas, entre ellos los órdenes Odonata, Dermaptera, Zoraptera, Phthiraptera, Psocoptera, Lepidoptera, Tricoptera y Hemiptera (Mola, 1995, Golub y Nokkala, 2004; Kuznetsova, Grozeva, Nokkala, 2004; Gavrilov, 2007; Papeschi y Bressa, 2006; Thakur y Gautam, 2013; Pita et al., 2017). Dentro de los arácnidos se encuentran representados en el grupo de los escorpiones en la familia Buthidae, en las arañas en el orden Aranae en la superfamilia Dysderidae y orden Astigmata (ácaros) (Rodríguez Gil, Mola, Papeschi, Scioscia, 2002; Schneider, Zacaro, Pinto-Da-Rocha, Candido, Cella, 2009). Los cromosomas holocinéticos también son frecuentes en nemátodos y en plantas de las familias Juncaceae, Cyperaceae y Cuscutaceae (Pazy, 1997; Pazy y Plitmann, 1991, 1994; García, 2001; García y Castroviejo, 2003; Mola, Rebagliati, Rodriguez Gil, Adilardi, 2011; Andrada, Páez, Toranzo, Ruíz de Bigliardo, 2018; Fogelberg, 1938; Oliveira et al., 2020).

Los Dermaptera, comúnmente llamados “tijeretas”, es uno de los órdenes que poseen cromosomas holocinéticos (Mola et al., 2011). Su distribución cosmopolita y su alimentación omnívora, son dos características que les atribuyen un gran valor como controladores de plagas (Brindle y Quintero Arias, 1992; Mariani, 1996, 1998; Romero Sueldo y Virla, 2005, 2009; Romero-Sueldo, Benítez de Parra, Torres de la Plaza, 2005; Romero Sueldo, Bruzzone, Virla, 2010). Dentro del género Doru (Forficulidae) encontramos a Doru linerae (Eschs.) y D. luteipes (Scudder) ambas especies de amplia distribución en Sudamérica alcanzando registros hasta el noroeste argentino 
(NOA) (Reichart, 1971; Mariani, 1996, 1998; Romero Sueldo y Virla, 2005, 2009; Romero Sueldo, 2012). En Tucumán, D. lineare es frecuente en los cultivares de maíz donde es un importante depredador de huevos de Spodoptera frugiperda Walker y de hemípteros auquenorrincos (Mariani, 1996). Mientras que D. luteipes, además de ser un depredador de plagas en cultivos de maíz, está presente en plantaciones de algodón y de caña de azúcar, en esta última consume huevos de Diatraea saccharalis Fabr. (Romero Sueldo y Virla, 2009).

Andrada, Silenzi-Usandivaras, Bigliardo, Romero, Dode (2016) permitieron determinar que $D$. lineare presenta $2 \mathrm{n}=20$ cromosomas holocinéticos y un mecanismo de determinación del sexo del tipo XX: XY (Q/O'). Además, estos autores reportaron que la especie puede presentar numerosas irregularidades meióticas. No se registraron datos citogenéticos para $D$. luteipes. Por tal motivo, el objetivo de este trabajo fue estudiar citogenéticamente a $D$. luteipes y realizar un análisis comparativo entre este taxón con $D$. lineare.

\section{MATERIALES Y MÉTODOS}

Para cada una de las especies analizadas, se recolectaron 30 machos adultos presentes en cultivos de maíz, ubicados en diferentes localidades: para $D$. lineare El Cadillal (Dpto. Tafí Viejo, Tucumán, $26^{\circ} 40^{\prime} 48^{\prime \prime}$, $65^{\circ} 16^{\prime} 00^{\prime \prime W}$ ) y para D. luteipes El Manantial

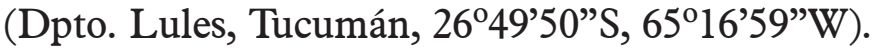

Los individuos se fijaron en solución de Farmer (alcohol etílico-ácido acético, 3:1) por $48 \mathrm{hs,} \mathrm{para} \mathrm{luego} \mathrm{ser} \mathrm{conservados} \mathrm{en} \mathrm{alcohol} 70 \%$ a $-4^{\circ} \mathrm{C}$ hasta su posterior utilización. Se emplearon dos técnicas citogenéticas de tinción; una clásica y otra con fluorocromo DAPI. Para la primera técnica, 15 gónadas de cada especie, fueron sumergidas durante $3 \mathrm{~min}$. en $\mathrm{HCl} 1 \mathrm{~N} \mathrm{a} 60^{\circ} \mathrm{C}$, posteriormente se lavaron en agua destilada y se les realizó la tinción con una gota de hematoxilina propiónica al $2 \%$ con cristales de citrato férrico. Para la técnica con fluorocromo DAPI, en ambas especies, se procesaron 15 folículos testiculares con una gota de ácido acético al $45 \%$ mediante el método de aplastado bajo cubreobjetos ("squash"). Posteriormente los preparados se congelaron con nitrógeno líquido, se removieron los cubreobjetos y a continuación se dejaron secar los portaobjetos a temperatura ambiente $\left(25^{\circ} \mathrm{C}\right)$ durante 24 hs; estos se colorearon con la técnica propuesta por Schweizer (1976), que utiliza como fluorocromo 4'6-diamidino-2-fenilindol (DAPI).

Para ambas especies analizadas, la longitud total de los cromosomas fue cuantificada en siete placas metafásicas mediante el programa Micromeasure 3.3 (Reeves, 2001). El análisis del comportamiento meiótico se realizó sobre un mínimo de 100 células por estadio.

Las imágenes se capturaron mediante una cámara Olympus QColor 5, acoplada a un microscopio de epifluorescencia Olympus BX43 equipado con una apropiada combinación de filtros. Los gráficos y las figuras se realizaron y se editaron, respectivamente, mediante el programa Corel Draw X3. 


\section{RESULTADOS}

\section{Mitosis}

En Doru lineare (Fig. 1 A) y D. luteipes (Fig. 1 D) se observó un complemento cromosómico diploide $2 \mathrm{n}=20$ (Fig. $1 \mathrm{~B}, \mathrm{E}$ ), con cromosomas carentes de constricciones primarias que se ubicaron paralelamente a los husos en mitosis y un sistema de determinación del sexo XX:XY (Q/ $\left./ \sigma^{\pi}\right)$. En $D$. lineare, los cromosomas sexuales heteromórficos se caracterizaron por tener el cromosoma $\mathrm{X}$ de mayor tamaño que el cromosoma Y (1,7 $\mu \mathrm{m}$ y $1,6 \mu \mathrm{m}$, respectivamente) y los cromosomas autosómicos variaron gradualmente de tamaño desde $3,75 \mu \mathrm{m}$ a $1,85 \mu \mathrm{m}$ (Fig. 1 C), con longitud del complemento cromosómico haploide de 27,34 $\mu \mathrm{m}$ (Tabla 1).

En tanto que en $D$. luteipes el cromosoma $\mathrm{X}$ también presentó mayor tamaño que el cromosoma $\mathrm{Y}(2,1 \mu \mathrm{m}$ y $1,8 \mu \mathrm{m}$, respectivamente) y los cromosomas autosómicos variaron desde $4,3 \mu \mathrm{m}$ a $2,3 \mu \mathrm{m}$ (Fig. $1 \mathrm{~F}$ ); la longitud de su complemento cromosómico haploide fue de $28,9 \mu \mathrm{m}$ (Tabla 1).

\section{Meiosis}

Ambas especies presentaron una fórmula haploide $\mathrm{n}=10(9 \mathrm{~A}+\mathrm{XY})($ Fig. 2, B; 3, B). Durante las etapas tempranas de la meiosis se observó heteropicnosis positiva de los cromosomas sexuales o fenómeno de alociclia (Fig. 2, A; Fig. 3, A).

Tabla 1. Parámetros morfométricos de los cromosomas de Doru lineare y D. luteipes. c= Longitud total. $\mathrm{CCH}=$ Complemento cromosómico haploide. $\mathrm{X}=$ Cromosoma sexual femenino. $\mathrm{Y}=$ Cromosoma sexual masculino. DE = Desvío estándar

Table 1. Morphometric parameters of the Doru lineare and D. luteipes chromosomes. $\mathrm{c}=$ Total length. $\mathrm{CCH}=$ haploid chromosome complement. $\mathrm{X}=$ female sex chromosome. $\mathrm{Y}=$ Male sex chromosome. SD = standard deviation.

\begin{tabular}{ccc}
\hline & \multicolumn{2}{c}{$\frac{\mathrm{c}(\mu \mathrm{m})}{\mathrm{X} \pm \mathrm{DE}}$} \\
\cline { 2 - 3 } Par cromosómico & Doru lineare & Doru luteipes \\
\hline 1 & $3,75 \pm 0,11$ & $4,30 \pm 0,10$ \\
\hline 2 & $3,15 \pm 0,10$ & $3,65 \pm 0,15$ \\
\hline 3 & $3,00 \pm 0,04$ & $3,25 \pm 0,05$ \\
\hline 4 & $3,00 \pm 0,06$ & $3,20 \pm 0,06$ \\
\hline 5 & $2,80 \pm 0,40$ & $2,75 \pm 0,30$ \\
\hline 6 & $2,65 \pm 0,10$ & $2,75 \pm 0,10$ \\
\hline 7 & $2,64 \pm 0,09$ & $2,65 \pm 0,12$ \\
\hline 8 & $2,55 \pm 0,10$ & $2,40 \pm 0,20$ \\
\hline$X$ & $1,85 \pm 0,12$ & $2,30 \pm 0,15$ \\
\hline$Y$ & $2,10 \pm 0,13$ & $1,70 \pm 0,80$ \\
\hline$C C H$ & $1,80 \pm 0,16$ & $1,60 \pm 0,11$ \\
\hline & 27,34 & 28,9 \\
\hline
\end{tabular}




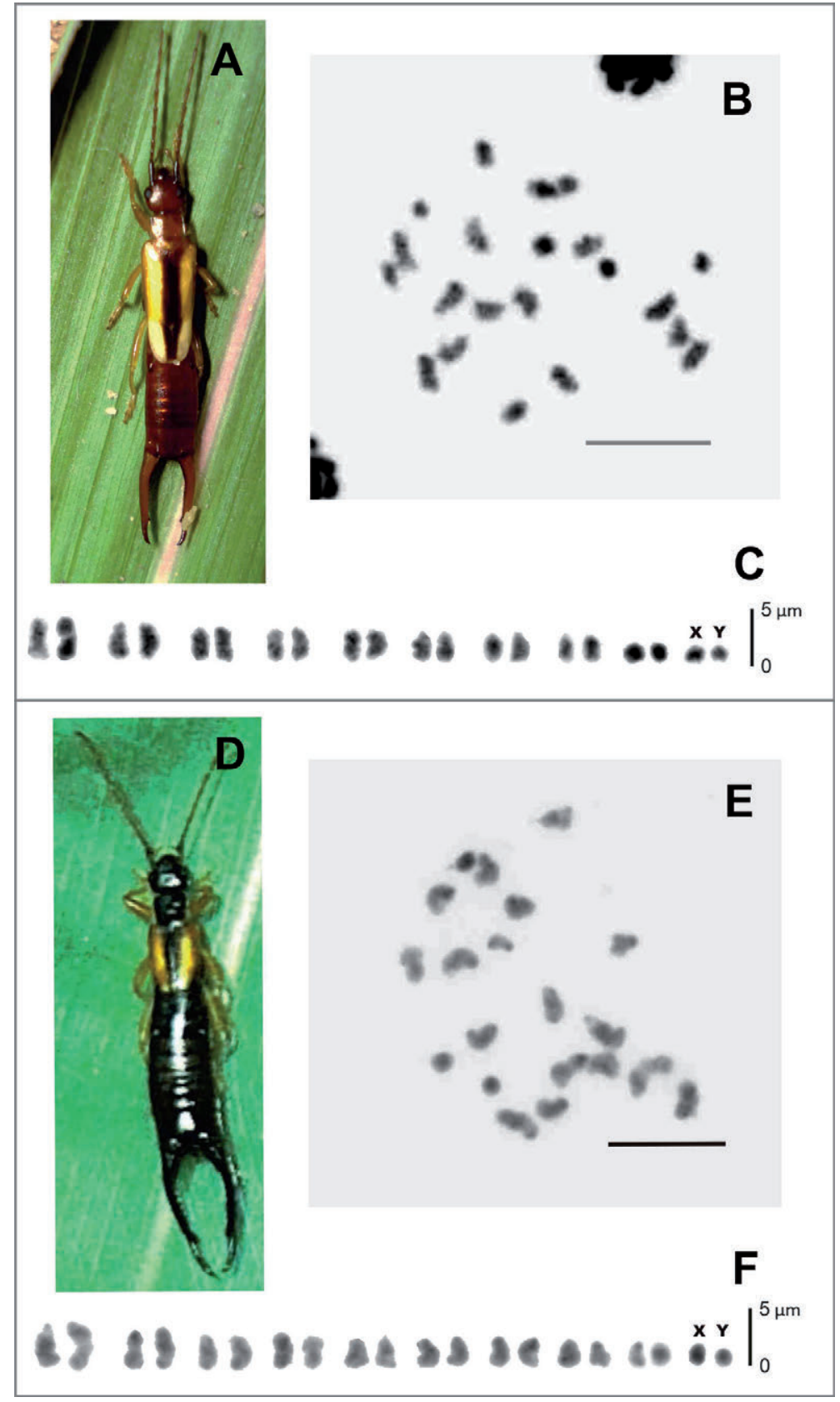

Figura 1. A-C: Doru lineare; D-F: D. luteipes. A y D vista general de un macho, B y E metafase mitótica $2 \mathrm{n}=20, \mathrm{C}$ y $\mathrm{F}$ cariograma.

Figure 1. A-C: Doru lineare; D-F: D. luteipes. A and D general view of a male, B and E mitotic metaphase $2 \mathrm{n}=20, \mathrm{C}$ and $\mathrm{F}$ karyogram.

En diacinesis, se observaron asociaciones secundarias no quiasmáticas en grupos de $4 / 6$ y $5 / 5$ en un $56 \%$ de las células en $D$. lineare (Fig. 2, D-E), mientras que en $D$. luteipes el $25 \%$ de las células presentaron asociaciones de 5 bivalentes (Fig. 3, C). A su vez en el $38 \%$ de las células de $D$. lineare exhibieron uno de los autosomas con constricción secundaria en un bivalente que le confiere un aspecto tripartito (Fig. 2, C-E). 

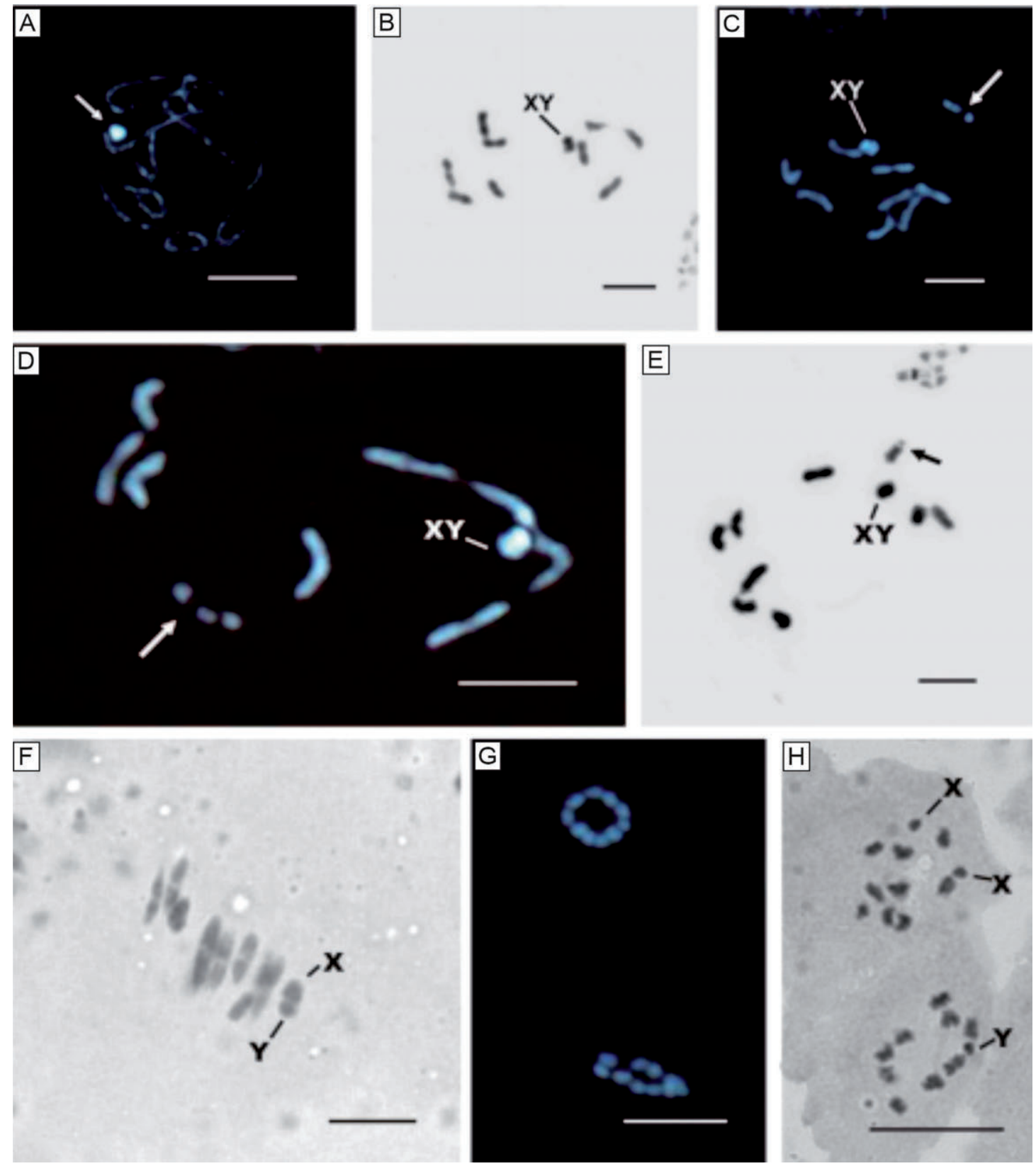

Figura 2. Doru lineare. A) Paquitene con cromosoma sexual condensado con heteropicnosis positiva. B) Diacinesis con $n=10$, los cromosomas sexuales heteromórficos. C) Diacinesis con autosoma de aspecto tripartito, con constricción secundaria. D) Asociación secundaria en diacinesis que incluyen el par $\mathrm{XY}$, se señala el autosoma con constricción secundaria. E) Diacinesis temprana con dos grupos polarizados de 5 cromosomas, se señala el autosoma con constricción secundaria. F) MI regular que exhibe el par sexual heteromórfico. G) Al con cromosomas conformando dos círculos. H) MII en vista polar con cromosomas $X$ disociado a modo de univalentes. Escala $=5 \mu \mathrm{m}$.

Figure 2. Doru lineare. A) Pachytene with a condensed sex chromosome with positive heteropycnosis. B) Diakinesis with $n=10$, heteromorphic sex chromosomes. C) Diakinesis with autosomal tripartite appearance, with secondary constriction. D) Secondary association in diakinesis including the XY pair, the autosome with secondary constriction is indicated. E) Early diakinesis with two polarized groups of 5 chromosomes, the autosome with secondary constriction is indicated. F) Regular Ml exhibited by the heteromorphic sex pair. G) Al with chromosomes forming two circles. H) MII in polar view with $\mathrm{X}$ chromosomes dissociated into univalents. Scale $=5 \mu \mathrm{m}$. 

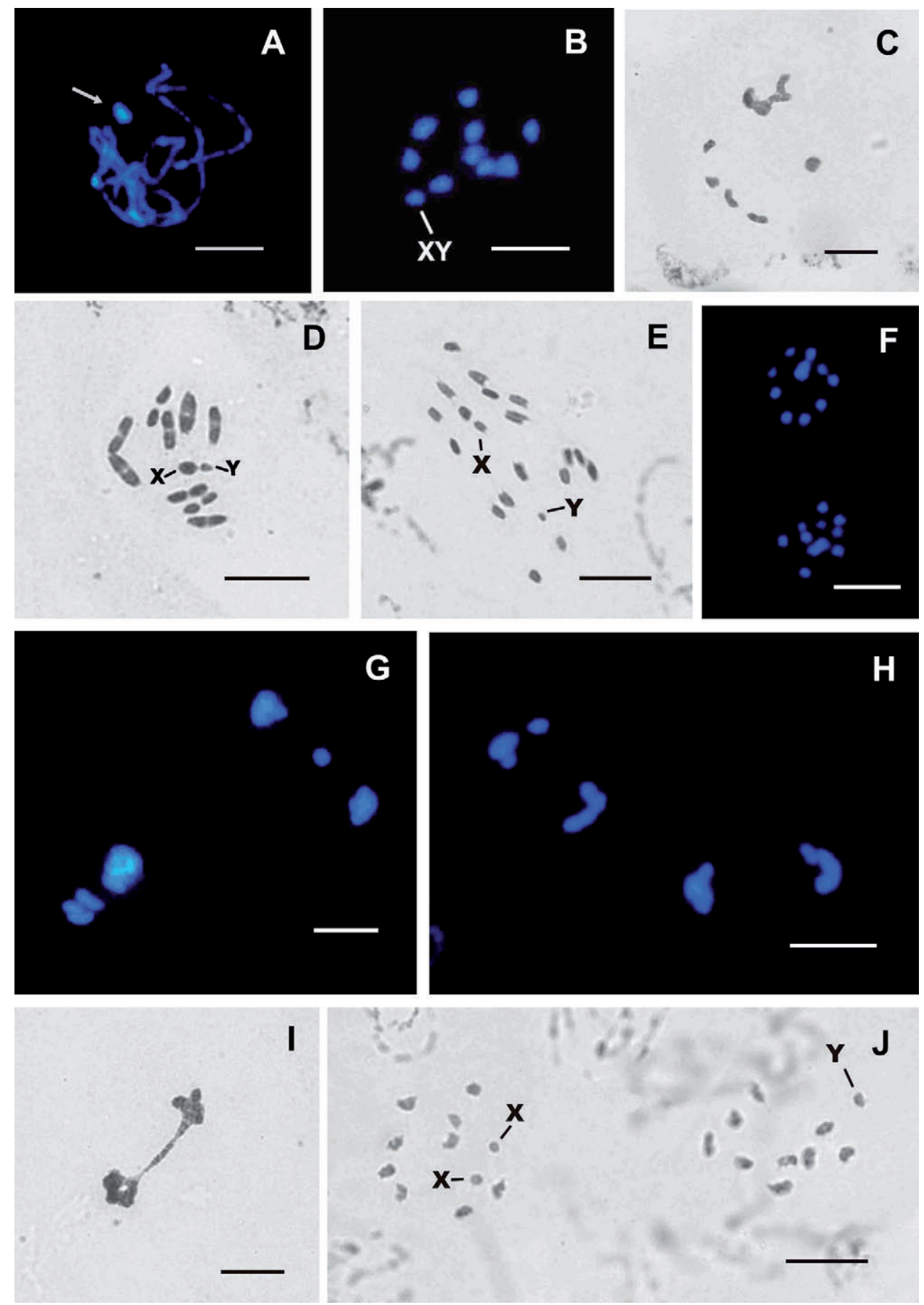

Figura 3. Doru luteipes. A) Paquitene con cromosoma sexual condensado con heteropicnosis positiva. B) Diacinesis con $n=10$ y cromosomas sexuales heteromórficos. C) Asociación cromosómica secundaria en diacinesis. D) MI con $n=10$, se muestra el par sexual heteromórfico XY. E) Al con segregación asincrónica de sus cromosomas. F) Al con cromosomas conformando un círculo en uno de los polos y un cromosoma central. G-H) MII con asociaciones cromosómicas secundarias. I) Al con puente de cromatina. J) MII con los cromosomas X disociados a modo de univalentes. Escala $=5 \mu \mathrm{m}$.

Figure 3. Doru luteipes. A) Pachytene with condensed sex chromosome with positive heteropycnosis. B) Diakinesis with $n=10$ and heteromorphic sex chromosomes. C) Secondary chromosomal association in diakinesis. D) MI with $\mathrm{n}=10$, the $\mathrm{XY}$ heteromorphic sex pair is shown. E) Al with asynchronous segregation of its chromosomes. F) Al with chromosomes forming a circle at one of the poles and a central chromosome. G-H) MII with secondary chromosomal associations. I) Al with chromatin bridge. J) MII with the X chromosomes dissociated into univalents. Scale $=5 \mu \mathrm{m}$. 
En ambas especies las Metafases I (MI) son regulares y sus cromosomas autosómicos constan de actividad cinética de tipo telocinética, segregando en la primera división ecuacionalmente, mientras que el par sexual heteromórfico se separa de modo reduccional durante Anafase I (AI) (Figs. 2 F, 3 D). En AI, D. lineare presentó el $80 \%$ de las células con los cromosomas dispuestos en forma de círculos y en el 10\% de los casos, se observó un patrón de migración a cada polo con uno o dos cromosomas en la región central del anillo de cromosomas (Fig. 2 G), mientras que en $D$. luteipes se observaron uno o dos cromosomas con disposición central en el 36\% de las AI (Fig. 3 F). Además, en esta etapa el 7\% de las células presentaron una segregación sincrónica de los cromosomas quedando en diferentes alturas de los husos (Fig. 3 E) y en menor medida (5\%) exhibieron puentes de cromatina (Fig. 3 I). La Telofase I (TI) fue regular.

En la segunda etapa de la división meiótica, en D. lineare el $45 \%$ de las Metafases II (MII) exhibieron células con el bivalente sexual XX a modo de dos univalentes (Fig. 2 $\mathrm{H})$, mientras que en $D$. luteipes se observó la misma situación, pero con un valor ligeramente mayor de 48\% (Fig. $3 \mathrm{~J}$ ). A su vez, en esta última especie durante MII, también se observaron asociaciones secundarias en dos grupos de 5 cromátidas (Fig. 3, G-H). Las Anafases II (AII) y Telofases II (TII) se desarrollaron de modo regular.

\section{DISCUSIÓN}

Dermaptera es un orden de insectos con cromosomas holocinéticos cuyos números cromosómicos son muy variados y poco estudiados. Se caracterizan por tener rangos de complementos diploides que van desde $2 \mathrm{n}=7$ (Arixeniidae) hasta $2 \mathrm{n}=60$ (Hemimeridae) (Jarvis, Haas, Whiting, 2004). Los estudios citogenéticos realizados por Andrada et al. (2016) revelaron números cromosómicos gamético $\mathrm{n}=10$ y somático $2 \mathrm{n}=20$ para $D$. lineare, los cuales coinciden con los observados en la presente investigación para la misma especie, como así también para los recuentos cromosómicos de $D$. luteipes. Cabe destacar que ambas especies presentan cromosomas holocinéticos y un sistema determinante del sexo de tipo XX:XY.

Los cromosomas holocinéticos han surgido de manera independiente varias veces durante la evolución tanto de plantas y como de animales (Mola y Papeschi, 2006) y, a pesar de su origen polifilético, presentan características comunes entre los diferentes grupos (Guerra et al., 2010); durante la mitosis presentan características únicas: 1- las metafases mitóticas con ausencia de constricciones primaria, 2- las cromátidas hermanas se ubican paralelas al plano ecuatorial en el estadio mitótico de la anafase, 3- cuando se fragmentan los segmentos cromosómicos persisten y se segregan durante la división celular (por ejemplo, cuando son irradiados con rayos $\mathrm{X}$, 4 la función cinetocórica no está centralizada sino que presentan placas proteicas que se extienden a lo largo de una gran superficie en cada cromátida (Mola y Papeschi, 2006); 5- mientras que en meiosis los cromosomas holocinéticos tienen autosomas con orientación de las cromátidas y patrones de migración de las mismas únicos, durante la primera división meiótica se produce la separación de cromátidas hermanas y la división se denomina post-reduccional (o ecuacional), mientras que 
en la segunda etapa de la división se produce la separación de segmentos homólogos y la división se designa reduccional (Mola y Papeschi, 2006).

Las especies estudiadas presentaron las características de los cromosomas holocinéticos: 1- tanto Doru lineare como D. luteipes carecen de constricción secundaria, 2- durante la anafase mitótica se ubican paralelamente a los husos, 3- cuando se fragmentan, sus segmentos se segregan de modo regular (lo que habría originado los meiocitos supernumerarios reportados como células poliploides por Andrada et al., 2016), 4 - la actividad cinética no está centralizada sino que se localiza en una gran región de los telómeros (telocinéticos), 5- presentan meiosis post-reduccional.

La holocentricidad de los cromosomas de ambas especies coincide con la naturaleza holocinética de los cromosomas establecida para el resto de los dermápteros (Giles y Webb, 1972; White, 1972; Mittal y Suri, 1981; Avancini, Recco-Pimentel, Prado, 2000; Gavrilov, 2007; Melters, Paulius, Korf, Chan, 2012). Sin embargo, como fue reportado en los cromosomas de $D$. lineare por Andrada et al., (2016) y lo observado en estas investigaciones para $D$. luteipes, estos cromosomas presentan zonas claras y oscuras que les brindan el aspecto de monocinéticos. Dicha coloración diferencial de la cromatina estaría relacionada a diferentes grados de condensación de la misma. Diferentes grados de condensación de la cromatina también se han encontrado en cromosomas politénicos de Drosophila melanogaster Meigen 1830, donde se diferencian en dominios topológicamente asociados (TADs, por sus siglas en inglés) que corresponden a bandas de cromatina densamente empaquetadas con actividad de transcripción limitada, mientras que las regiones entre los TADs (inter-TADs) se corresponden a inter-bandas descondensadas de estos cromosomas densamente activas (Ulianov et al., 2016). Tal vez en el género Doru, los meiocitos necesitan mantener activos algunos genes durante la división celular y, de este modo, parte de la cromatina permanece descondensada para tal fin, confiriéndole a los cromosomas la diferencia de tinción antes descripta.

Las asociaciones secundarias son un indicio de que los genomas que dieron origen al organismo o al individuo, a pesar de estar muy emparentados entre sí, son lo suficientemente diferentes como para que los cromosomas no homólogos (provenientes de un mismo parental) se asocien de manera inespecífica y no quiasmática, mediante segmentos heterocromatínicos que se atraen entre sí, como lo describe Kuznetsova, Golup, Aguin Pombo (2013). Sumado a las asociaciones secundarias, generalmente en grupos de 5 cromosomas, la falta de homología observada entre los cromosomas X en Doru lineare y en D. luteipes, los cuales se disocian como univalentes durante la MII, sugieren que el género podría haber tenido un origen híbrido. La disociación temprana de los cromosomas X en las dos especies de Doru analizadas, durante la segunda división, agregan sustento a la aseveración que los cromosomas sexuales tienen segregación reduccional durante AI y se mantienen unidos como bivalentes por medio de los "crossing over" durante la MI, mientras que en las MII estos permanecen separados por causa de la ausencia de entrecruzamientos y la carencia de homología de los segmentos $\mathrm{X}$ segregantes.

Chirino y Bressa (2014) observaron en prometafases meióticas del hemíptero Belostoma candidulum Montandon, 1903 (Heteroptera: Belostomatidae) constricciones secundarias en los cromosomas X. Estos autores hacen mención a que dichas 
constricciones tendrían una función cinética durante la fijación de los cromosomas a las fibras del huso. Constricciones secundarias similares fueron observadas en $D$. lineare, sin embargo, éstas no estaban presentes en los cromosomas sexuales sino en uno de los bivalentes autosómicos; las mismas fueron interpretadas anteriormente por Andrada et al., (2016) como fragmentación de cromosomas.

Para tener una visión más amplia de la función que cumplen estos cambios estructurales en los cromosomas, es necesario realizar estudios con técnicas adicionales, por ejemplo, estudios moleculares donde se pueda determinar la distribución de las proteínas del cinetocoro. Uno de los principales componentes proteicos es la proteína centromérica CENP (Melters et al., 2012), su funcionalidad y modo de organización en los cromosomas brindaría información adicional que podría contribuir al estudio del comportamiento y evolución de los cromosomas hasta alcanzar el holocentrismo, tal como ocurre en estos insectos.

También es necesario incrementar el conocimiento genético en las especies aún no analizadas que son afines al género y así, poder tener un indicio de la función cinética que cumplirían las mencionadas constricciones secundarias.

\section{CONCLUSIONES}

Se reportan por primera vez recuentos cromosómicos $\mathrm{n}=9+\mathrm{XY}$ y $2 \mathrm{n}=18+$ $\mathrm{XX} / \mathrm{YY}$ para $\mathrm{O}^{\prime \prime}$ de $D$. luteipes. Las especies aquí estudiadas comparten: la presencia de cromosomas holocinéticos con el orden Dermaptera, ambas especies presentan cromosomas sexuales heteromórficos y el mismo tipo de irregularidades. Por otro lado, estas especies se diferencian en la longitud de sus cromosomas, que se pone de manifiesto en el mayor tamaño del complemento cromosómico haploide de $D$. luteipes. Entre las anormalidades podemos mencionar la alta frecuencia de asociaciones cromosómicas secundarias y la disociación del bivalente sexual $\mathrm{X}$ en la segunda división meiótica, que hace inferencia a que este género podría tener un posible origen híbrido.

\section{AGRADECIMIENTOS}

A la Fundación Miguel Lillo por su constante apoyo y a los revisores por sus valiosas sugerencias.

\section{FINANCIAMIENTO}

El viaje y los recursos utilizados fueron financiados por la Fundación Miguel Lillo a través del proyecto CUP B-0013-1 del Instituto de Genética de la Fundación Miguel Lillo. 


\section{PARTICIPACIÓN}

Todos los autores participaron de manera equitativa en la elaboración del trabajo.

\section{CONFLICTO DE INTERESES}

Declaramos explícitamente que no existen conflictos de intereses entre los autores

\section{LITERATURA CITADA}

Andrada, A. R., Páez, V. A., Toranzo, M. I., Ruíz de Bigliardo, G. E. (2018). Análisis de la meiosis y viabilidad del polen en morfotipos intraespecíficos de Cuscuta parodiana (Cuscutaceae). Lilloa, 55 (1), 3 15. https://doi.org/10.30550/ j.lil/2018.55.1

Andrada, A. R., Silenzi Usandivaras, G. M., Bigliardo, G. E., Romero, M., Dode, M. (2016). Estudio citogenético en dos poblaciones naturales de Doru lineare (Dermáptera, Forficulidae). Acta zoológica Lilloana, 60 (1), 3-9. http://www. lillo.org.ar/journals/index.php/acta-zoologica-lilloana/article/view/99

Avancini, R. M. P., Recco-Pimentel, S. M., Prado, A. (2000). Labidura riparia (Dermaptera, Labiduridae) from Brazil: Karyotype and C- banding. Cytologia, 65 (1), 93-96.https://doi.org./10.1508/cytologia.65.93

Brindle, A., Quintero Arias, D. (1992). Earwigs of Panama. In: Insects of Panama and Mesoamerica. Selected Studies. D. Quintero Arias and A. Aiello (Eds). Oxford: Oxford University Press, $692 \mathrm{pp}$.

Chirino, M. G., Bressa, M. J. (2014). Karyotype evolution in progress: A new diploid number in Belostoma candidulum (Heteroptera: Belostomatidae) from Argentina leading to new insights into its ecology and evolution; Czech Academy of Sciences; European Journal of Entomology (Ceske Budejovice), 111 (2), 165-174. http://dx.doi.org/10.14411/eje.2014.027

García, M. A. (2001). A new Western Mediterranean species of Cuscuta (Convolvulaceae) confirms the presence of holocentric chromosomes in subgenus Cuscuta. Botanical Journal of the Linnean Society 135: 169-178. https://www.research gate.net/deref/http\%3A\%2F\%2Fdx.doi.org\%2F10.1111\%2Fj.1095-8339.2001. tb01089.x

García, M. A., Castroviejo S. (2003). Estudios citotaxonómicos en las especies ibéricas del género Cuscuta (Convolvulaceae). Anales del Jardín Botánico de Madrid, 60 (1): 33-44.

Gavrilov, I. A. (2007). A catalogue of chromosomal numbers and genetic systems of scale insects (Homoptera: Coccinea) of the world. Israel Journal of Entomology, 37, 1-53.

Giles, E. T., Webb, G. C. (1972). The Systematics and karyotype of Labidura truncata Kirby, 1903 (Dermaptera: Labiduridae). Australian Journal of Entomology, 11, 253-256. https://doi.org/10.1111/j.1440-6055.1972.tb01625.x 
Golub, N., Nokkala, S. (2004). Chromosome numbers of two sucking louse species (Insecta, Phthiraptera, Anoplura). Hereditas, 141, 94-96. https://doi.org/10.1111/ j.1601-5223.2004.01859.x

Guerra, M., Cabral, G., Cuacos, M., González-García, M., González-Sánchez, M., Vega, J., Puertas, M. J. (2010). Neocentrics and holokinetics (holocentrics): chromosomes out of the centromeric rules. Cytogenetic and Genome Research, 129, 82-96. https:// doi.org/ 10.1159/000314289

Hughes-Schrader, S., Ris, H. (1941). The diffuse spindle attachment of coccids verified by the mitotic behavior of induced chromosome fragments. Journal of Experimental Zoology, 87, 429-456. https://doi.org/10.1002/jez.1400870306

Jarvis, K. J., Haas, F., Whiting, M. F. (2004). A phylogeny of earwigs (Insecta: Dermaptera) based on molecular and morphological evidence: reconsidering the classification of Dermaptera. Systematic Entomology, 30, 1-12. https://doi. org/10.1111/j.1365-3113.2004.00276.x

Kuznetsova, V. G., Grozeva, S., Nokkala, S. (2004). New cytogenetic data on Nabidae (Heteroptera: Cimicomorpha), with a discussion of karyotype variation and meiotic patterns, and their taxonomic significance. European Journal of Entomology, 101 (2), 205-210. https://doi.org/10.14411/eje.2004.026

Kuznetsova, V. G., Golup, N., Aguin Pombo, D. (2013). Karyotypes, B-chromosomes and meiotic abnormalities in 13 populations of Alebra albostriella and A. wahlbergi (Hemiptera, Auchenorrhyncha, Cicadellidae) from Greece. Comparative Cytogenetics, 7, 305-325. doi//.org/ 10.3897/CompCytogen.v7i4.6411

Mariani, R. (1996). Catálogo de insectos fitófagos argentinos (Dermaptera). XX International Congress of Entomology, Proceedings Taxonomy of the Dermaptera, Firenze (Italia), 386 pp.

Mariani, R. (1998). Dermaptera. En: J. J. Morrone y S. Coscarón (eds.), Biodiversidad de Artrópodos Argentinos, Una Perspectiva Biotaxonómica, Ediciones Sur, La Plata, Argentina, pp. 35-47

Melters, D. P., Paulius, L. V., Korf, I. F., Chan, S. W. L. (2012). Holocentric chromosomes: convergent evolution, meiotic adaptations, and genomic analysis. Chromosome Research, 20, 579-593. https//doi.org/ 10.1007/s10577-012-9292-1

Mittal, O. P., Suri, V. (1981). Chromosome studies in two species of earwings (Dermaptera: Diplatyidae) from India. Caryologia, 34, 441-446. https//doi.org/10.3897/ CompCytogen.v7i4.6411

Mola, L. M. (1995). Post reductional meiosis in Aeshna (Aeschnidae, Odonata). Hereditas, 122, 47-55. http://dx.doi.org/10.1111/j.1601-5223.1995.00047.x

Mola, L. M., Papeschi, A. G. (2006). Holokinetic chromosomes at a glance. BAG Journal of Basic and Applied Genetics, 17-33. http://hdl.handle.net/20.500.12110/ paper_16660390_v17_n1_p17_Mola

Mola, L. M., Rebagliati, P. J., Rodriguez Gil, S. G., Adilardi, R. S. (2011). Variaciones meióticas y evolución cromosómica en insectos y arácnidos con cromosomas holocinéticos. Journal of Basic and Applied Genetics, 22, 1-6.

Oliveira, L., Neumann, P., Jang, T-S., Klemme, S., Schubert, V., Koblížková, A., Houben, A, Macas, J. (2020). Mitotic spindle attachment to the holocentric 
chromosomes of Cuscuta europaea does not correlate with the distribution of CENH3 chromatin. Frontiers in Plant Science, 10, 1779-1799.

Papeschi, A. G., Bressa, M. J. (2006). Evolutionary Cytogenetics in Heteroptera. Journal of Biological Research, 5, 3-21.

Pazy, B. (1997). Supernumerary chromosomes and their behaviour in meiosis of the holocentric Cuscuta babylonica Choisy. Botanical Journal of the Linnean Society 123: 173-176. https://doi.org/10.1111/j.1095-8339.1997.tb01411.x

Pazy, B., Plitmann, U. (1991). Unusual chromosome separation in meiosis of Cuscuta L. Genome 34: 533-536. http://dx.doi.org/10.1139/g91-082

Pazy, B., Plitmann, U. (1994). Holocentric chromosome behaviour in Cuscuta (Cuscutaceae). Plant Systematic Evolution191: 105-109. https://doi.org/10.1007/ BF00985345

Pita, S., Lorite, P., Vela, J., Mora, P., Palomeque, T., Thi, K. P., Panzera, F. (2017). Holocentric chromosome evolution in kissing bugs (Hemiptera: Reduviidae: Triatominae): diversification of repeated Sequences. Parasites \& Vectors 10:410. https://doi.org/10.1186/s13071-017-2349-4

Reeves, A. (2001). MicroMeasure: a new computer program for the collection and analysis of cytogenetic data. Genome 44: 439-443. http://dx.doi.org/10.1139/g01037

Reichart, H. (1971). Catologue of New Word Dermaptera Forficuloidea. Papeis Avulsos Zoologia, 24 (4), 161-184.

Rodríguez Gil, S., Mola, L., Papeschi, A., Scioscia, C. (2002). Cytogenetic heterogeneity in common haplogyne spiders from Argentina (Arachnida, Araneae). The Journal of Arachnology 30: 47-56. http://dx.doi.org/10.1636/0161-8202

Romero Sueldo, M., Benítez de Parra, L., Torres de la Plaza, M. I. (2005). Duración y supervivencia de los estadios ninfales de Doru lineare (Dermáptera: Forficulidae) sometidos a diferentes dietas. Acta Zoológica Lilloana, 49, 59-63.

Romero Sueldo, M., Virla, E. (2005). Forage preference of Doru lineare (Eschs.) (Dermaptera: Forficulidae) under laboratory conditions. Revista de la Sociedad de Entomología Argentina, 64, 430-431.

Romero Sueldo, M., Virla, E. G. (2009). Doru lineare (Dermaptera: Forficulidae), insecto benéfico en cultivos de maíz del norte argentino: preferencias alimenticias y tasas de consumo. Boletín de Sanidad Vegetal- Plagas, 35, 39-47. http://hdl. handle.net/11336/47329

Romero Sueldo, M., Bruzzone, O. A., Virla, E. G. (2010).Characterization of Doru lineare (Eschscholtz) (Dermaptera - Forficulidae) as predator of fallarmy worm larvae (Lep. - Noctuidae): a functional response study. Journal of Insect Science, 10, 1536-2442. https://doi.org/10.1673/031.010.3801

Romero Sueldo, G. M. (2012). Evaluación de Doru lineare (Dermaptera, Forficulidae) como depredador de Spodoptera frugiperda (Lepidoptera, Noctuidae. Editoral Académica Española, Saarbrucken, Alemania, 1-75.

Schneider, M. C., Zacaro, A. A., Pinto-Da-Rocha, R., Candido, D. M., Cella, D. M. (2009). A comparative cytogenetic analysis of two Bothriuridae species and overview of the chromosome data of scorpions. Journal of Heredity, 100, 545555. https://doi.org/10.1159/000445863 
Schweizer, D. (1976). Reverse fluorescent chromosome banding with chromomycin and DAPI. Chromosoma 58: 307-324. https://doi.org/10.1007/BF00292840

Thakur, R., Gautam, D. C. (2013). Chromosome Studies on Four Species of Moths. Cytologia, 78 (3), 327-331. https://doi.org/10.1508/cytologia.78.327

Ulianov, S. V.; Khrameeva, E. E., Gavrilov, A. A., Flyamer, I. M., Kos, P., Mikhaleva, E. A., Penin, A. A., Logacheva, M. D., Imakaev, Chertovich, M. V. A., Gelfand M. S., Shevelyov, Y. Y., Razin, S. V. (2016). Active chromatin and transcription play a key role in chromosome partitioning into topologically associating domains. Genome Research, 26, 70-84. https//doi:10.1101/gr.196006.115

White, M. J. (1972). The chromosomes of Arixenia essau Jordan (Dermaptera). Chromosoma, 36, 338-342. https://doi.org/10.1007/BF00336792 